\title{
Fault Classification of Rolling Bearing Based on Time-Frequency Generalized Dimension of Vibration Signal and ANFIS
}

\author{
Fang $\mathrm{Li}^{*}$
}

School of Electric Multiple Units Engineering, Dalian Jiaotong University, No.794, Huanghe road, Shahekou District, Dalian, Liaoning province, 116028, China

\begin{abstract}
Research shows that multi-fractal can not only exhibit the singular probability distribution form of the fractal signal completely, but also increase the fine level of signal geometrical characteristics and local scaling behavior. Based on multi fractal dimension calculation of time-frequency matrix of vibration signal of rolling bearing in this paper, energy distribution characteristics of time-frequency domain of vibration signal could be extracted, then adaptive fuzzy neural network (ANFIS) was used in signal classification. Experiments showed that this method can realize fault classify of rolling bearing effectively, it is feasible in engineering application.
\end{abstract}

Keywords: Classify, multi-fractal, time frequency matrix, vibration.

\section{INTRODUCTION}

The mechanical fault diagnosis technology is the identification science of the machine or equipment operation state. It is composed of 4 steps: signal detection, feature extraction, condition recognition and diagnosis decision, in which feature extraction is more important. A fault feature may contain several fault information [1], while one of fault information may be reflected in several fault features. The result of mutual penetration and mutual cross make it more difficult in equipment fault diagnosis. Although several features may reflect the same fault, but reflection sensitivity of fault state is different, so it is necessary to choice the appropriate feature to use as fault diagnosis criterion. In geometry respects fractal reflect hierarchical structure of things, while fractal reflects hierarchical structure of process in time respects. The significance of fractal dimension is as follow: It describes degree of dissipation energy in nonlinear system, the fractal dimension is greater, the dissipation energy is more, the fractal dimension is smaller, and the dissipation energy is less. The dissipation system will degenerate into conservative system once the fractal dimension is zero. There are many monitor methods in mechanical fault diagnosis field. But owing to the complexity of fault, sub harmonic vibration and the same frequency vibration are not enough, it is necessary to consider the more complex vibration characteristics. In recent years, fractal which as a fault feature describe tool, has been applied in fault diagnosis $[2,3]$.

For nonlinear and non stationary reciprocating mechanical vibration, it requires understanding for local information of signal. It couldn't tell us what time a certain frequency component will occur if only for analysis in time domain, while it is very important for non stationary signal. This paper presents a method, first of all to fractal vibration signal in time domain, then extract feature of signal, finally realize fault classify of rolling bearing combination ANFIS.

\section{NUMERICAL ALGORITHM OF THE GENERALIZED DIMENSION IN TIME-FREQUENCY DOMAIN [4]}

Given time series $X_{n}$ : after EMD decomposition and instantaneous frequency transform of $X_{n}$, the results are as follows:

$J(\omega, t)=\sum_{i=1}^{n} b_{i} A_{i}(t) e^{j \omega_{i}(t) t}$

After normalization the amplitude matrix $\mathrm{A}_{\mathrm{UV}}$ could be obtained, every point in it correspond to a time and instantaneous frequency, the square of the point represent the amount of time and instantaneous frequency normalization energy in time and frequency domain.

Similar to division of sample space of time domain signal, take $\varepsilon_{j}$ as width of grid, $\mathrm{J}$ as the number of data points of grid, which is species number. Then The number of rows and columns in the grid are $m=F / \varepsilon_{j}, n=T / \varepsilon_{j}$. Suppose grid of $m$ row and $\mathrm{n}$ column as $\mathrm{mn}$ grid [5], record energy of mn Network coverage collection as $E_{m n}$, then energy distribution probability of grid Network coverage collection:

$$
P=\left(\varepsilon_{j}\right)=\frac{E_{m n}}{\sum_{m=1}^{R} \sum_{n=1}^{P} E_{m n}}=E_{m n} / E
$$


By the above formula a series of $K_{q}\left(\varepsilon_{j}\right)$ could be obtained.

$$
\text { Let } T(j)=\log \left(\varepsilon_{j}\right), S_{j}=K_{q}\left(\varepsilon_{j}\right) \text {, then }
$$

$S(j)=-D_{q} T(j)+B$

Gradient of $\mathrm{Dq}$ is general dimension, $\mathrm{B}$ is intercept, and then function is as follow:

$f\left(D_{q}, B\right)=\sum_{j=1}^{J}\left[S(j)+D_{q} T(j)-B\right]^{2}$

It can be seen from least square method, the function has a minimum value condition is:

$\frac{\partial f}{\partial D_{q}}=0, \frac{\partial f}{\partial D_{q}}=0$

The result is as follow:

$$
\begin{aligned}
D_{q}^{E}\left(\varepsilon_{j}\right)= & \frac{J \sum_{j=1}^{J} T(j) S(j)-\sum_{j=1}^{J} S(j) \sum_{j=1}^{J} T(j)}{J \sum_{j=1}^{J} T(j)^{2}-\left[\sum_{j=1}^{J} T(j)\right]^{2}} \\
= & \frac{J \sum_{j=1}^{J} K_{q}\left(\varepsilon_{j}\right) \lg \varepsilon(j)-\sum_{j=1}^{J} \lg \varepsilon(j) \sum_{j=1}^{J} K_{q}\left(\varepsilon_{j}\right)}{J \sum_{j=1}^{J}\left(K_{q}\left(\varepsilon_{j}\right)\right)^{2}-\left[\sum_{j=1}^{J} K_{q}\left(\varepsilon_{j}\right)\right]^{2}}
\end{aligned}
$$

\section{ADAPTIVE FUZZY NEURAL INFERENCE NETWORK (ANFIS)}

Adaptive Fuzzy Neural Inference System was put forward by Chinese scholars Jyh-Skiing Roger Jang in 1993; it is an integration system combination neural network and fuzzy theory. The system use topology structure of neural network to express fuzzy inference system, realize various fuzzy operations by each neuron, adjust system parameter adaptive with the hybrid algorithm to error back propagation and least squares estimation in neural network theory, to approach implication relation between input and output data. The system has the functions of fuzzy reasoning and mapping approximation ability. It has been applied in system identification, time series prediction and fault diagnosis and so on $[6,7,8,9]$.

\subsection{Structure of ANFIS}

ANFIS is a multi input single output system, without loss of generality. For example, Surgeno fuzzy system, suppose there are two input $x, y$ and one output $\mathrm{f}$, for a first order Sugeno, typical fuzzy ruler expression is:

$$
\begin{aligned}
& \text { If } x \text { is } A_{1} \text { and } y \text { is } B_{1} \text { then } f_{1}=p_{1} x+q_{1} y+r_{1} . \\
& \text { If } x \text { is } A_{2} \text { and } y \text { is } B_{2} \text { then } f_{2}=p_{2} x+q_{2} y+r_{2} \text {. }
\end{aligned}
$$

The corresponding ANFIS structure is as shown in Fig. (1). In Fig. (1), the line between nodes only represents direction, without weight related; parameters can be adjusted in square node, while parameters are fixed circle node.

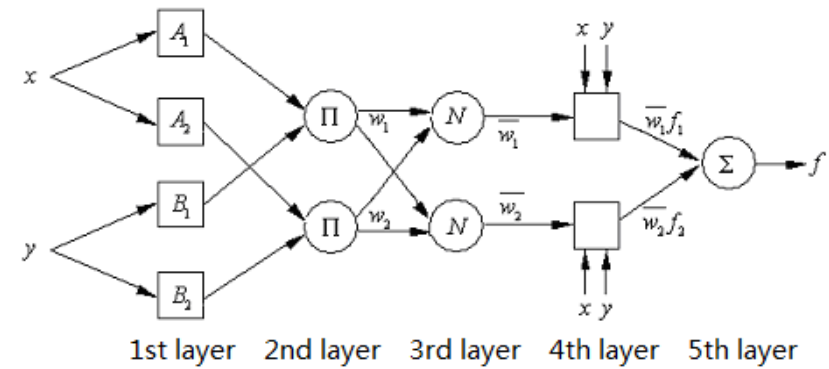

Fig. (1). Structure of ANFIS.

\subsection{ANFIS Learning Algorithm}

In ANFIS, system variable parameters are adjusted by the hybrid algorithm to error back propagation and least squares estimation. The linear parameters could be identified by least squares method and nonlinear parameters could be identified by error back propagation in adjustable parameter adaptive networks.

\section{FAULT CLASSIFY BASED ON TIME-FREQUENCY GENERALIZED DIMENSION AND ANFIS}

Multi-component singular spectrum analysis method has advantages of nonlinear signals, non stationary signals with local wave method, decomposes the signals into the basic mode component according to from high frequency to low frequency, then executes the phase space reconstruction and singular entropy calculation. The singular entropy of each component, which used as input parameters, were input the ANFIS reasoning system to realize fault classify. It retains advantages that nonlinear, non stationary was decomposed into single component by local wave analysis as well as restructure the dynamic system, so the original dynamic system was analyzed on basis of multi component. The specific process is shown in Fig. (2):

\section{APPLICATION EXAMPLES}

Bearing test machine is shown in Fig. (3). A large number of bearing vibration data was obtained after long term field monitoring. The data analysis frequency is 10 $\mathrm{kHz}$, the intercepted length of training and inspecting data is 1024 time point, as shown in the Fig. (4):

Suppose $j=1,2,3,4$, represent normal state, inner ring fault, outer ring fault, retainer fault respectively. There are 120 groups of data, 80 groups of data were used as training sample. In 4 states, the group's number of each state is 20; the other 40 group could be used as the test samples, the groups number of each state is 10 .

To avoid there are too many dimensions, the first 5 dimension data in every group was calculated. So there are 5 input variables in system, every input section was divided into 4 parts. Therefore the combination of input variables and the rules obtained a total of 45 .

After data training with ANFIS neural network, inspect results were shown as Tables $\mathbf{1}$ and $\mathbf{2}$. 


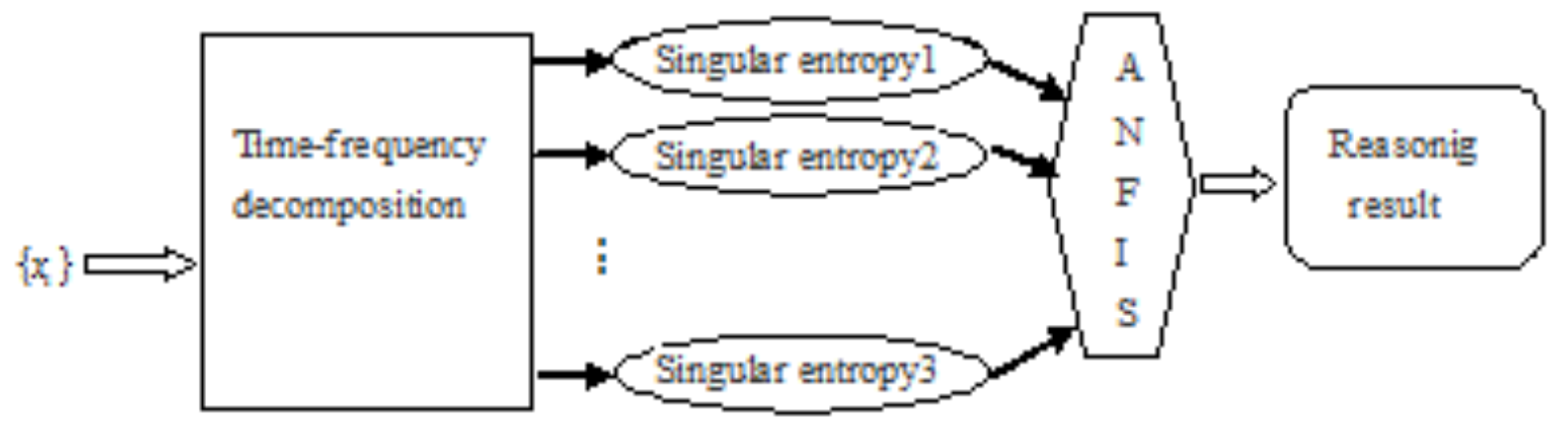

Fig. (2). Classify model.
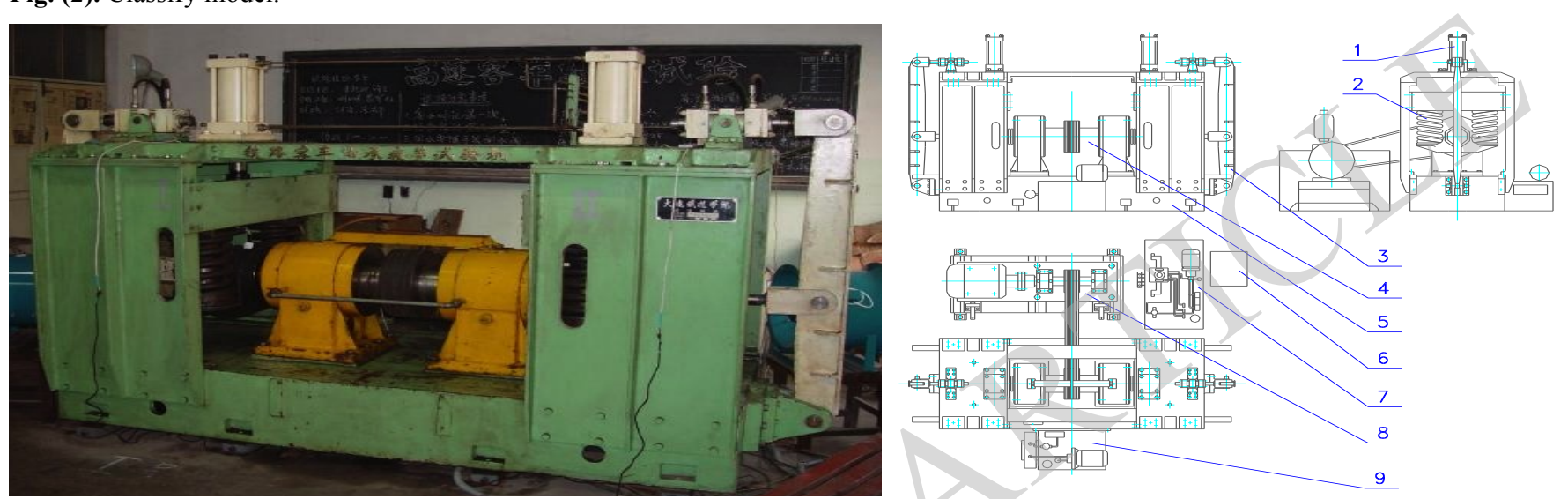

1 Radial loading device 2 Axle box 3 Axial loading device 4 Main shaft of testing machine 5 Base of test machine 6 Electrical control cabinet 7 Hydraulic station 8 Transmission 9 Cooling system

Fig. (3). Bearing test machine.

The result shows that the method of adopting 1 Analysis of 10 groups, including the wrong divided into two groups, the remaining 8 groups correctly from the above analysis result, so the classify accuracy of this method is high and it is feasible in practice.

\section{CONCLUSION}

The generalized fractal dimension of time domain and frequency domain could reflect various working state of rolling bearing well, it is easy and feasible to realize fault mode identified without disintegration by the

Table 1. Output of method 1 by ANFIS (1).

\begin{tabular}{|c|c|c|c|c|c|c|}
\hline Signal & $D_{1}$ & $\mathbf{D}_{2}$ & $\mathbf{D}_{3}$ & $\mathbf{D}_{4}$ & $\mathbf{D}_{5}$ & Category \\
\hline 1$)$ & -2.9162 & -1.9355 & -2.0444 & -1.8474 & -1.8470 & 1 \\
\hline 2 & -2.8829 & -2.0310 & -2.0001 & -1.9216 & -1.9017 & 1 \\
\hline 3 & -2.5277 & -2.0333 & -1.9573 & -1.9033 & -1.7425 & 1 \\
\hline 4 & -2.9033 & -2.1010 & -1.9999 & -1.9023 & -1.8700 & 1 \\
\hline 5 & -5.8000 & -5.3440 & -5.3440 & -4.8702 & -4.8126 & 2 \\
\hline 6 & -5.8553 & -5.2851 & -5.2475 & -5.0324 & -4.8006 & 2 \\
\hline 7 & -5.9013 & -5.3272 & -5.0881 & -4.9030 & -4.8999 & 2 \\
\hline 8 & -6.0231 & -5.5462 & -4.9965 & -4.9027 & -4.8553 & 2 \\
\hline 9 & -8.3260 & -7.9957 & -7.6545 & -7.5233 & -7.7851 & 3 \\
\hline 10 & -8.7454 & -8.5252 & -8.0332 & -7.5949 & -7.5949 & 3 \\
\hline 11 & -8.5466 & -8.0325 & -8.0027 & -7.5326 & -7.4227 & 3 \\
\hline 12 & -8.7901 & -8.3527 & -8.1147 & -7.9696 & -7.5532 & 3 \\
\hline 13 & -1.7142 & -1.2721 & -1.0357 & -0.7081 & -0.6999 & 4 \\
\hline 14 & -2.0352 & -1.95487 & -1.5426 & -1.5424 & -1.2395 & 4 \\
\hline
\end{tabular}


generalized dimension of time domain and frequency domain was used as characteristic value.

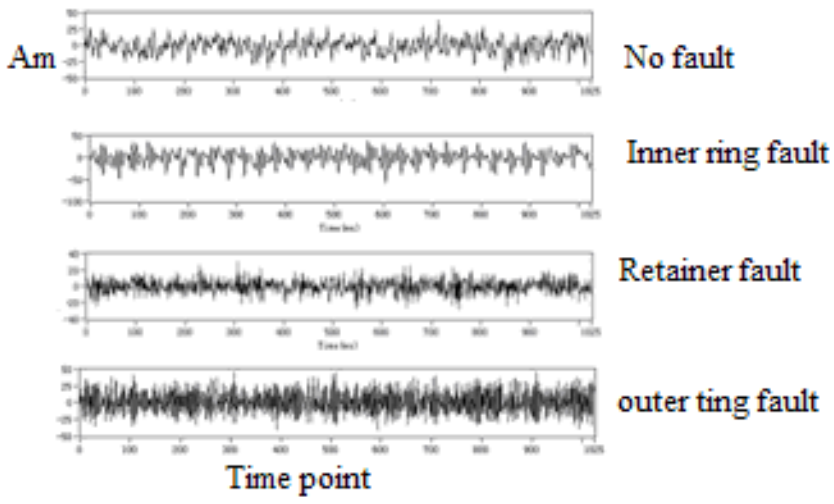

Fig. (4). Waveform.

This paper use the time-frequency dimension method of bearing vibration signal as the input of adaptive fuzzy neural network to realize classify of fault of rolling bearing. The results show that the classification of the accuracy of this method is high, and it has certain application value.

Table 2. Output of method 1 by ANFIS (2).

\begin{tabular}{|c|c|c|c|c|}
\hline State & State 1 & State 2 & State 3 & State 4 \\
\hline \hline state 1(10 group) & 10 & 0 & 0 & 0 \\
\hline state 2(10 group) & 0 & 10 & 0 & 0 \\
\hline state 3(10 group) & 1 & 0 & 9 & 0 \\
\hline state 4(10 group) & 0 & 0 & 1 & 9 \\
\hline
\end{tabular}

Revised: December 15, 2014

Accepted: December 16, 2014

\section{CONFLICT OF INTEREST}

The authors confirm that this article content has no conflict of interest.

\section{ACKNOWLEDGEMENTS}

This work is supported by the Education Department of Liaoning Province, China (No. L2014187).

\section{REFERENCES}

[1] B. Shi, and Y. Shen. "The fractal method in mechanical fault diagnosis", Beijing, Metallurgical Industry Press, 1989.

[2] Z. Xu, L. Fang, X. Wang etc. Beijing, National Defense Industry Press, 2000.

[3] Yuxiu. Xu, J. Zhong, and B. Wen, "Fractal characteristics and dynamic characteristics of the rotating machinery fault diagnosis", Chinese Journal of Mechanical Engineering, vol. 41, no. 12, pp. 186-189, 2005.

[4] Y. Yu, Z. Xing, and Y. Zhao, "A new time-frequency analysis method, the local wave method", Test and diagnosis of vibration, vol. 13, pp.219-224, 2000.

[5] R. J. Jyh-Shing, "ANFIS: adaptive-network-based fuzzy inference system", IEEE Transactions on Systems, vol. 23, no. 3, pp. 665$685,1993$.

[6] R. J. Jyh-Shing, and C.T. Sun, "Nero-fuzzy modeling and control", In: Proceedings of the IEEE, vol.83, no.3, pp. 378-406, 1995.

[7] T.G.B. Amaral, M. Manuel, and P. Fernao, "Adaptive system for modeling and control", In: Proceedings of First International Intelligent Systems, vol. 1, pp. 67-72, 2002.

[8] C. S. G. Lee, and J. S. Wang, "Self-adaptive neuro-fuzzy systems: structure and learning", In: Proceedings of the 2000 IEEE/RSJ International Conference on Robots and Systems, pp. 52-57, 2000.

[9] J. S. Wang, and C. S. G. Lee, "Self-adaptive neuro-fuzzy inference systems for classification applications", In: IEEE Transactions on Fuzzy Systems, vol.10, no.6, pp. 790-802, 2002.
Received: December 8, 2014

(C) Fang Li; Licensee Bentham Open.

This is an open access article licensed under the terms of the Creative Commons Attribution Non-Commercial License (http://creativecommons.org/licenses/by-nc/3.0/) which permits unrestricted, non-commercial use, distribution and reproduction in any medium, provided the work is properly cited. 